\title{
Dry-spot nucleation in thin liquid films on chemically patterned surfaces
}

\author{
Berend J. Brasjen • Anton A. Darhuber
}

Received: 21 March 2011 / Accepted: 21 June 2011/Published online: 19 July 2011

(C) The Author(s) 2011. This article is published with open access at Springerlink.com

\begin{abstract}
We systematically study the influence of chemical patterning on the instability of thin liquid films induced by chemical heterogeneities on a flat, horizontal, and partially wetting substrate. We consider common geometric shapes like wedges, circles, and stripes and determine the time required for nucleation of a dry-spot as a function of film thickness, contact angle, pattern dimensions, and geometry. Moreover, we characterized the resulting liquid distribution and identified conditions that avoid the formation of residual droplets on the less wettable regions, which is usually undesirable in technological applications.
\end{abstract}

Keywords Dewetting - Chemically patterned substrates . Thin liquid films · Disjoining pressure

\section{Introduction}

Hydrophobic or partially wetting surfaces are relevant to a number of technological applications ranging from nonstick surface treatments (Moilliet 1963; Kissa 2001) to offset printing (Senefelder 1911; Kipphan 2001; Darhuber et al. 2001). Often they are applied if no liquid entrainment on a substrate or certain regions on it is desired. In painting technology, the opposite situation frequently arises, i.e., a

B. J. Brasjen · A. A. Darhuber $(\bowtie)$

Mesoscopic Transport Phenomena Group, Department

of Applied Physics, Eindhoven University of Technology,

Den Dolech 2, 5612AZ Eindhoven, The Netherlands

e-mail: a.a.darhuber@tue.nl

B. J. Brasjen

Dutch Polymer Institute (DPI), P. O. Box 902,

5600 AX Eindhoven, The Netherlands partially wetting surface should be coated with a stable uniform liquid film that dries or solidifies without defect formation. An instability of a liquid film of initially uniform thickness can induce the nucleation of dry-spots that subsequently grow in size, which is commonly summarized under the term dewetting phenomena.

Sheludko (1967), Vrij (1966), and Vrij and Overbeek (1968) considered attractive Van der Waals forces as the rupture mechanism of free-standing liquid films such as soap lamellae and pointed out an analogy with phase separation by spinodal decomposition (Cahn 1965). Ruckenstein and Jain (1974) performed a stability analysis of thin liquid films on a solid substrate and extended the analysis towards the presence of soluble and insoluble surfactants. The same authors treated the case of liquid films adhering to a solid substrate and immersed in a second immiscible bulk liquid phase and elucidated the effect of viscosity ratio and surface viscosity on the rupture time scale (Jain and Ruckenstein 1976). Gumerman and Homsy (1975) examined the effects of film drainage and the presence of lateral sidewalls and found a critical thickness much smaller than Ruckenstein and Jain (1974). Williams and Davis extended the previously considered linear stability theories towards non-linear effects that become dominant in the late stages of the rupture process (Williams and Davis 1982). Sharma and Ruckenstein (1986) determined the stability of spatially non-homogeneous stationary solutions and found a faster rate of thinning and shorter dominant wavelength as compared to the linear theory. Burelbach et al. (1988) considered the instability of volatile, evaporating or condensing liquid films.

Brochard Wyart and Daillant (1990) and Redon et al. (1991) studied the hole growth dynamics after nucleation of a dry-spot in a thin liquid film. Brochard Wyart et al. (1993) investigated liquid-liquid dewetting. Buguin et al. 
(1999) and Noblin et al. (2006) studied inertial effects. Reiter (1992; Reiter 1993; Reiter et al. 2005) and Xie et al. (1998) investigated the dewetting of ultrathin films of polymer melts on Si wafers. The effect of slip at the solidliquid interface on the formation and growth of dry patches was studied by Seemann et al. (2001), Sharma and Kargupta (2003), Kargupta et al. (2004), and Fetzer et al. (2005). Karthaus (1999) observed the formation of ordered arrays of micrometer-sized polymer arrays upon dewetting of a polystyrene solution on mica, $\mathrm{Si}$, and glass substrates.

Sharma (1993) and Sharma and Jameel (1993) considered in addition to the well-studied apolar, Lifshitz-Van der Waals interactions also polar, acid-base interactions, and specifically the breakup of aqueous films (Sharma 1998), which have been studied experimentally by Stöckelhuber (2003; Stöckelhuber et al. 2004). Thiele et al. (2001a, b) extended the study of Sharma and Ruckenstein (1986) and elucidated the role of non-uniform initial conditions on the rupture dynamics of unstable thin liquid films. Sharma et al. (2002) studied the influence of density variations and effective compressibility on film breakup. Verma et al. (2006) investigated pattern formation in thin liquid films confined between two plates. Kalpathy et al. (2010) showed that shear in a liquid bilayer sandwiched between parallel plates can delay or even suppress rupture.

Kargupta et al. systematically studied the dewetting dynamics of thin liquid films on substrates with a wide range of surface patterns, including single and periodic stripes, circles, squares, and square arrays. They systematically studied the effect of initial film thickness, pattern dimensions, and surface energy contrast (Kargupta et al. 2000; Kargupta and Sharma 2002; Sharma et al. 2003; Kargupta and Sharma 2003). Sehgal et al. (2002) and Zhang et al. (2003) performed experiments regarding the dewetting of polystyrene layers on periodically heterogeneous substrates. Thiele et al. considered the morphology evolution in a thin liquid film on substrates with one- and two-dimensional sinusoidal surface energy modulation (Brusch et al. 2002; Thiele et al. 2003; Pototsky et al. 2004). Kao et al. (2006) investigated the rupture of thin liquid films on substrates with sinusoidal and square-wave resonant surface energy patterning. Julthongpiput et al. (2007) studied the transition from isotropic to patterndirected dewetting on substrates with differential surface energy contrast. Zhu et al. (2010) fabricated binary surface topographies consisting of alternating ridges and droplet arrays by means of controllable dewetting. Koch et al. (1995) studied the morphology and line tension of ultrathin liquid films in the vicinity of a chemical step heterogeneity using a density functional approach, whereas Moosavi et al. (2008) investigated the motion of nanodroplets on similarly patterned substrates.
Many applications in organic electronics require precise liquid film deposition on chemically patterned surfaces. Two strategies for selective deposition onto patterned substrates are (i) applying the liquid exclusively to the desired regions (Biebuyck and Whitesides 1994; Darhuber et al. 2000; Brasjen et al. 2011) and (ii) coating the entire surface with an uniform film and relying on subsequent liquid redistribution (Bower et al. 2007). In the latter process, it is necessary that this redistribution is completed before the applied solution dries and desirable that no residual volume is left outside the designated regions. As a first step towards the characterization of the process window, we conducted numerical simulations for pure, nonvolatile fluids. We determined the critical film thickness above which no major redistribution occurs, the nucleation time for dry-spot formation, and the residual volume for geometrically simple and common surface energy patterns, such as halfplanes, wedges, circles, and stripes.

For this purpose, we systematically consider various disjoining pressure isotherms with destabilizing term of the form $A / h^{m}$, where $h$ is the film thickness and $A$ and $m$ are constants. This allows parametrization of arbitrary experimental disjoining pressure isotherms, at least over a finite range of film thicknesses. While several groups have already studied dewetting of thin liquid films on structured surfaces (Kargupta et al. 2000; Kargupta and Sharma 2002; Sharma et al. 2003; Kargupta and Sharma 2003; Sehgal et al. 2002; Zhang et al. 2003; Brusch et al. 2002; Thiele et al. 2003; Pototsky et al. 2004; Kao et al. 2006; Julthongpiput et al. 2007; Zhu et al. 2010), most of the results have been obtained for small film thicknesses on order of ten times the precursor layer thickness, very specific disjoining pressure isotherms with more than one inflection point or neglecting the influence of gravity. For industrial applications relevant film thicknesses range typically between 1 and $10 \mu \mathrm{m}$, which is several orders of magnitude above the precursor layer thickness such that the influence of gravity can be substantial if not decisive. We seek to answer the question up to what thickness liquid films can still be controlled and modulated by means of substrate patterning. Moreover, we systematically discuss the film evolution in terms of contact angles, which greatly aids the practical application and interpretation of our results.

\section{Theoretical model and numerical implementation}

In a long-wave (i.e., small slope) approximation, the time evolution of the film thickness $h$ on a flat, horizontally oriented, and chemically patterned substrate is governed by the lubrication equation (Oron et al. 1997)

$\frac{\partial h}{\partial t}-\frac{\partial}{\partial x}\left(\frac{h^{3}}{3 \mu} \frac{\partial p}{\partial x}\right)-\frac{\partial}{\partial y}\left(\frac{h^{3}}{3 \mu} \frac{\partial p}{\partial y}\right)=0$, 
in which

$p=-\gamma\left(\frac{\partial^{2} h}{\partial x^{2}}+\frac{\partial^{2} h}{\partial y^{2}}\right)+\rho g h-\Pi$

represents the augmented pressure (Oron et al. 1997), $\mu$ the viscosity, $\rho$ the density, and $\gamma$ the surface tension. The disjoining pressure $\Pi$ describes contributions to the effective pressure in ultrathin films that are absent or immeasurably small in thicker films or bulk volumina. These contributions can arise from a number of interactions such as Van der Waals forces, double layer attraction or repulsion as well as steric effects and molecular or supramolecular layering (Derjaguin et al. 1987). For instance in "black" soap films the disjoining pressure can be an oscillatory function of film thickness as a consequence of micellar layering (Bergeron and Radke 1992).

Schwartz and Eley (1998) introduced a phenomenological model for $\Pi$, which exhibits a single minimum but no local maximum

$\Pi_{\mathrm{SE}}=\gamma(1-\cos \theta) \frac{(n-1)(m-1)}{(n-m) h^{*}}\left[\left(\frac{h^{*}}{h}\right)^{n}-\left(\frac{h^{*}}{h}\right)^{m}\right]$.

Here, $n>m>1$ are positive real numbers, $\theta(x, y)$ is the equilibrium contact angle pattern, and $h^{*}$ is a length scale of typically few nanometers, at which $\Pi_{\mathrm{SE}}\left(h=h^{*}\right)=0$ holds. Such a power law representation of the disjoining pressure can be quantitatively accurate at least in a certain thickness range as for instance the disjoining pressure component due to non-retarded Van der Waals forces scales as $A / h^{3}$, whereas e.g., water on quartz gives rise to a disjoining pressure proportional to $h^{-2}$ or $h^{-1}$, depending on the film thickness range. (Derjaguin et al. 1987) The dependence of $\Pi_{\mathrm{SE}}$ on $h$ is plotted in Fig. 1 for different values of $h^{*}$. Smaller values of $h^{*}$ and larger values of $(n, m)$ generally increase $\left|\mathrm{d} \Pi_{\mathrm{SE}} / \mathrm{d} h\right|$ and make the system of Eqs. 1-3 stiffer. $\Pi_{\mathrm{SE}}$ has a single minimum and a single inflection point, located at $h_{\min }=\frac{n}{m} h^{*}$ and $h_{\text {ip }}=\frac{n(n+1)}{m(m+1)} h^{*}$,

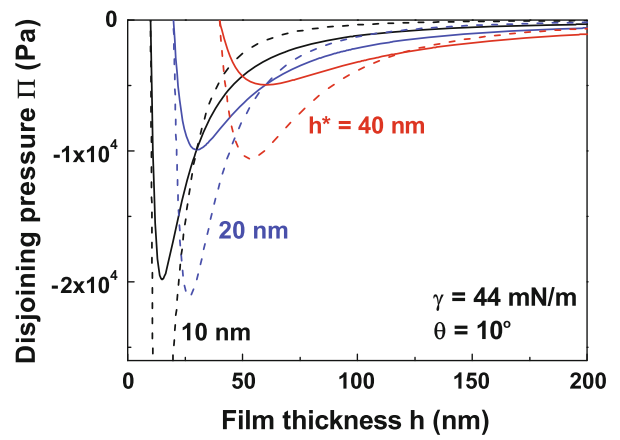

Fig. 1 Model disjoining pressure $\Pi(h)$ for $\gamma=44 \mathrm{mN} / \mathrm{m}, \theta=10^{\circ}$, and $(n, m)=(3,2)$ (solid lines) and $(n, m)=(4,3)$ (dashed lines) (a)
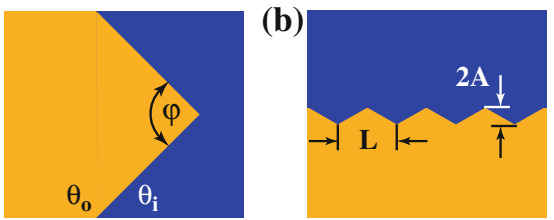

(c)

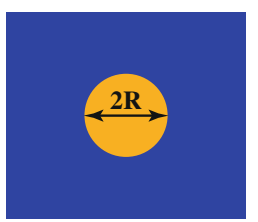

(d)

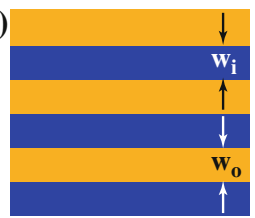

Fig. 2 [Color online] Sketch of chemical surfaces patterns studied in this manuscript: a wedges, $\mathbf{b}$ corrugated edges, $\mathbf{c}$ circles, and $\mathbf{d}$ stripe arrays. The blue (dark) and orange (light) colors indicate regions of the hydrophilic and hydrophobic equilibrium contact angles $\theta_{\mathrm{i}}$ and $\theta_{\mathrm{o}}$, respectively

respectively. As is evident from Eq. 3, a variation of $\theta$ modulates $\Pi_{\mathrm{SE}}$ by means of a multiplying factor.

We consider flat, horizontal surfaces with regions characterized either by the hydrophobic contact angle $\theta_{\mathrm{o}}$ or the hydrophilic contact angle $\theta_{i}$. Several elementary contact angle patterns $\theta(x, y)$ such as stripes, circles, and wedges are sketched in Fig. 2.

The set of Eqs. 1-3 is solved numerically using the finite element (FEM) software Comsol 3.5a in conjunction with Matlab 7.9.0. In all cases, the initial condition corresponded to a film of uniform thickness $h(x, y, t=0)=h_{0}$. Boundary conditions were either (1) symmetry conditions such as $\partial h / \partial x=0$ and $\partial p / \partial x=0$ or (2) a constant film thickness $h=h_{0}$ at boundaries sufficiently remote from the edges of the contact angle patterns such that they did not affect the dynamics in the interior of the computational domain during the simulated time interval. The lubrication approximation restricts the validity of the model to small slopes (Snoeijer 2006) and thus contact angles below approximately $30^{\circ}$.

In order to identify scales for non-dimensionalization of the evolution Eqs. 1 and 2, we briefly review the results of linear stability theory applied to liquid films on chemically homogeneous surfaces. These provide length- and timescales that are instrumental for the discussion of the dewetting dynamics on heterogeneous substrates.

\subsection{Chemically homogeneous surfaces}

Liquid films on homogeneous surfaces with contact angle $\theta_{\mathrm{o}}$ are absolutely stable for (Finn 1986) $h_{0}>h_{\mathrm{as}} \equiv$ $2 \ell_{\mathrm{c}} \sin \left(\frac{1}{2} \theta_{\mathrm{o}}\right)$, in which $\ell_{\mathrm{c}} \equiv \sqrt{\gamma /(\rho g)}$ is the capillary length of the liquid. Films are unstable for $h_{0}<h_{\mathrm{us}}$ or $\theta_{\mathrm{o}}>\theta_{\mathrm{us}}$, where the parameters $h_{\mathrm{us}}$ and $\theta_{\mathrm{us}}$ are defined by the condition $\frac{\mathrm{d} \Pi}{\mathrm{d} h}=\rho g$, and metastable inbetween these limits, $h_{\mathrm{us}}<h<h_{\mathrm{as}}$. For sufficiently large values of $n-m$ and 
$h_{\mathrm{us}} \gg h^{*}$, the term proportional to $h^{-n}$ in Eq. 3 is small and the following relations hold to good approximation

$h_{\mathrm{us}} \approx h^{*}\left(\frac{\gamma(1-\cos \theta)}{\rho g} \frac{(n-1)(m-1) m}{h^{* 2}(n-m)}\right)^{\frac{1}{m+1}}$

and

$1-\cos \left(\theta_{\mathrm{us}}\right) \approx \frac{\rho g}{\gamma} \frac{h^{* 2}(n-m)}{(n-1)(m-1) m}\left(\frac{h_{0}}{h^{*}}\right)^{m+1}$.

In this context, metastability denotes stability against infinitesimal disturbances but instability towards height disturbances of sufficient amplitude. Considering harmonic disturbances $h=h_{0}+h_{1} \cos (2 \pi x / \lambda)$ with amplitude $h_{1} \ll$ $h_{0}$ and wavelength $\lambda$ of the uniform base state $h=h_{0}$, the linear instability regime is characterized by a maximally unstable wavelength (Ruckenstein and Jain 1974; Williams and Davis 1982)

$\lambda_{\max }=2 \pi\left(\frac{2 \gamma}{-\rho g+\frac{\mathrm{d} \Pi}{\mathrm{d} h}\left(h_{0}\right)}\right)^{1 / 2}$

with corresponding growth rate

$$
\left(\frac{1}{h_{1}} \frac{\mathrm{d} h_{1}}{\mathrm{~d} t}\right)_{\max }=\frac{h_{0}^{3}}{12 \mu \gamma}\left[-\rho g+\frac{\mathrm{d} \Pi}{\mathrm{d} h}\left(h_{0}\right)\right]^{2} .
$$

The inverse growth rate can serve as a characteristic time scale for dry-spot nucleation. Disturbances of subcritical wavelength $\lambda<\lambda_{\mathrm{c}} \equiv \lambda_{\max } / \sqrt{2}$ decay due to the stabilizing action of surface tension.

Based on Eqs. 6 and 7, we choose the following nondimensional variables:

$\bar{t}=t / T, \quad$ where $\quad T \equiv 12 \mu \gamma h_{0}^{-3}\left[\frac{\mathrm{d} \Pi}{\mathrm{d} h}\left(h_{0}\right)\right]^{-2}$

$\bar{x}=x / \Lambda, \quad$ where $\quad \Lambda \equiv 2 \pi\left[\frac{1}{2 \gamma} \frac{\mathrm{d} \Pi}{\mathrm{d} h}\left(h_{0}\right)\right]^{-1 / 2}$

and $\bar{h}=h / h_{0}$.

\section{Results and discussion}

We systematically vary the pattern geometry from unbounded domains like adjacent half-planes of different surface energy and wedges to compact shapes such as circles and stripes, where confinement effects induced by an additional geometric length scale play a role. Exemplary simulations were performed for $h^{*}=10 \mathrm{~nm}, \theta_{\mathrm{i}}=0^{\circ}$ and the material parameters $\mu=58.3 \mathrm{mPa} \mathrm{s}, \gamma=44.0 \mathrm{mN} / \mathrm{m}$, and $\rho=1126 \mathrm{~kg} / \mathrm{m}^{-3}$, which corresponds to the model liquid tetra(ethylene glycol) with $\ell_{\mathrm{c}} \approx 2.0 \mathrm{~mm}$.

\subsection{Adjacent half-planes}

The most elementary chemical surface patterning corresponds to two halfplanes with different contact angles meeting each other at $x=0$. We will consider the case

$\theta(x, y)=\left\{\begin{array}{ccc}\theta_{\mathrm{o}} & \text { for } & x<0 \\ 0 & \text { for } & x \geq 0\end{array}\right.$

with the initial condition of a flat and uniform film thickness $h(x, t=0)=h_{0}$. The step change in $\Pi$ at $x=0$ induces pressure-driven flow from the partially wetting to the completely wetting side, which causes film thinning at the left side and accumulation at the right side of $x=0$, as shown in Fig. 3a. For sufficiently small $h_{0}$, the local depression in the height profile grows and after a time $\tau$ induces nucleation of a dry-spot.

Figure $3 b$ shows $\tau$, defined as the time at which the minimum value of $h$ corresponds to $h_{\mathrm{ip}}$, as a function of $1-\cos \theta_{\mathrm{o}}$ for two sets of exponents $(n, m)$ and three values of $h_{0}$. The solid lines correspond to power law relations $\tau \sim\left(1-\cos \theta_{\mathrm{o}}\right)^{-2}$. The dashed vertical lines represent the minimum unstable contact angle $1-\cos \theta_{\text {us }}$ for a given initial film thickness $h_{0}$, according to Eq. 5. In Fig. 3c is plotted the nucleation time $\tau$ as a function of $h_{0}$ for two values of $\theta_{\mathrm{o}}$ and two sets of exponents $(n, m)$. The solid lines correspond to power law relations $\tau \sim h_{0}^{2 m-1}$, whereas the dashed vertical lines indicate the maximum thickness for linear instability of a film on a homogeneous surface with contact angle $\theta_{\mathrm{o}}$ according to Eq. 4. Figure $3 \mathrm{~d}$ corresponds to a non-dimensional version of Fig. 3c, which indicates a divergence of $\tau / T$ at the same value of $h / h_{\mathrm{us}} \approx 1.36$ for all parameter values considered. Figure $3 \mathrm{e}$ presents the distance $d$ of the dry-spot nucleation point from the contact angle discontinuity at $x=0$ as a function of $h_{0}$ for $\theta_{\mathrm{o}}=10^{\circ}$ and two sets of exponents $(n, m)$. The solid lines correspond to power law relations $d \sim h_{0}^{(m+1) / 2}$.

The step geometry is invariant under a coordinate transformation $x \rightarrow x / a$ with $a$ an arbitrary positive number, provided that the origin of the coordinate system $x=0$ coincides with the location of the contact angle discontinuity. For sufficiently thin films, $h_{0} \ll h_{\mathrm{us}}$, the influence of gravity can be neglected for the film conformation and dynamics in the vicinity of the step, i.e., as long as the height perturbation is confined to the region $|x| \lesssim \ell_{\mathrm{c}}$. Moreover, if $h_{0} \gg h^{*}$, then the term proportional to $h^{-n}$ in Eq. 3 is also negligible in the initial phase of dry-spot formation. The remaining terms in Eq. 1 are invariant under the transformations $(h, x, t) \rightarrow(h / b, x / a, t / c)$, if the parameters are chosen according to $a=b^{m+1}$ and $c=b^{2 m-1}$. The last relation provides a rigorous explanation for the behavior $\tau \sim$ $h_{0}^{2 m-1}$ for $h^{*} \ll h_{0} \ll h_{\mathrm{us}}$ found in Fig. 3 b. 

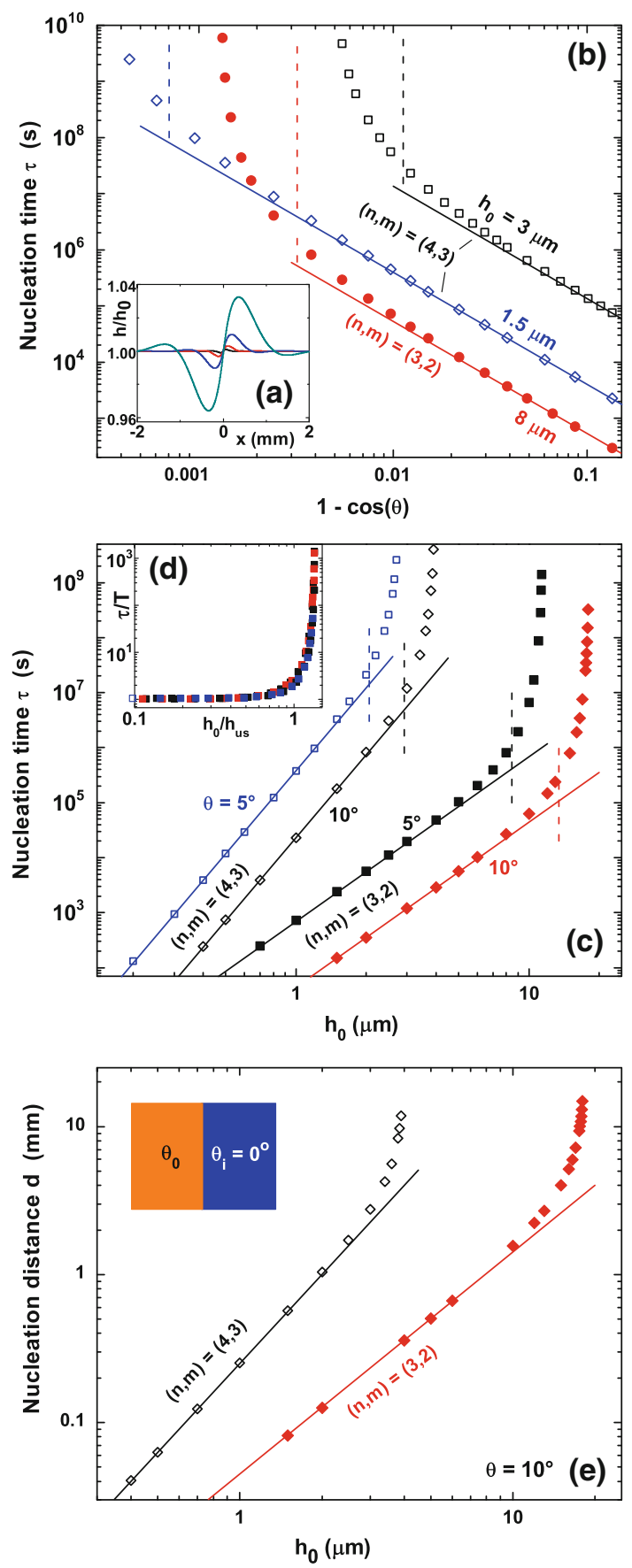

Fig. 3 a Time evolution of the normalized height profile $h(x, t) / h_{0}$ near the step discontinuity for $h_{0}=1.5 \mu \mathrm{m}, \theta_{\mathrm{o}}=10^{\circ}$ and $(n, m)=(3,2)$. b Nucleation time $\tau$ at a step discontinuity in contact angle as a function of $1-\cos \theta_{\mathrm{o}}$ for $(n, m)=(4,3)$ (open symbols) and $(n, m)=(3,2)$ (solid symbol) and three values of $h_{0}$. The solid lines correspond to power law relations $\tau \sim\left(1-\cos \theta_{0}\right)^{-2}$, the dashed vertical lines correspond to Eq. 4. c Nucleation time $\tau$ at a step discontinuity in contact angle as a function of $h_{0}$ for $(n, m)=(4,3)$ (open symbols) and $(n, m)=(3,2)($ solid symbols $)$ and two values of $\theta_{\mathrm{o}}=5^{\circ}$ and $10^{\circ}$. The solid lines correspond to power law relations $\tau \sim h_{0}^{2 m-1}$, the dashed vertical lines correspond to Eq. 4. d Non-dimensional version of c. e Distance $d$ of dry-spot nucleation point from contact angle discontinuity as a function of $h_{0}$ for $\theta_{\mathrm{o}}=10^{\circ}$ and two sets of exponents $(n, m)$. The solid lines correspond to power law relations $d \sim h_{0}^{(m+1) / 2}$
The power law relations $\tau \sim h_{0}^{2 m-1}$ (solid lines in Fig. 3b) and $\tau \sim\left(1-\cos \theta_{0}\right)^{-2}$ (solid lines in Fig. 3c) are consistent with $\tau \sim T$ as defined in Eq. 8 after dropping the first term in $\Pi_{\mathrm{SE}}$ involving the larger exponent $n$. The latter omission is permissible given $n>m$ and $h \gg h^{*}$ during the stage of the nucleation process that effectively determines its timescale $\tau$.

The power law relations $d \sim h_{0}^{(m+1) / 2}$ (solid lines in Fig. 3e) are consistent with $d \sim \Lambda$ as defined in Eq. 9, again after dropping the first term in $\Pi_{\mathrm{SE}}$ involving the larger exponent $n$. According to Fig. 3a, the separation between the local minimum and maximum in the film thickness progressively increases. Once it corresponds to the critical wavelength $\lambda_{\mathrm{c}}=\lambda_{\max } / \sqrt{2}$, the linear instability mechanism accelerates the deepening of the depression. Therefore, a first-order estimate might be $d \approx \lambda_{\mathrm{c}} / 4 \approx 0.18 \lambda_{\max }$. The fitted power law relations in Fig. 3d correspond to $d \approx$ $0.125 \lambda_{\max }$ for $m=2$ and $d \approx 0.147 \lambda_{\max }$ for $m=3$.

These scaling relations show that the dry-spot nucleation process adjacent to a step discontinuity in the contact angle follows the same scaling laws as on a homogeneous surface. It is, however, a deterministic process in the sense that it will proceed even for a perfectly flat initial film profile and that the position of the (first) dry-spot is given by $d$ and, thus, known a priori.

The presence of the chemical discontinuity destabilizes films of thickness $h_{0}$ just above the metastability limit for homogeneous surfaces of contact angle $\theta_{\mathrm{o}}$. From Fig. $3 \mathrm{~b}$, we estimated that the metastability limit for step discontinuities is higher than $h_{\mathrm{us}}$ given in Eq. 4 by a factor $1.36 \pm 0.01$, which appears to be independent of $n, m$ and $\theta_{\mathrm{o}}$ (Fig. 3d). We attribute this increase in destabilization strength to the non-infinitesimal nature of the perturbation caused by the contact angle discontinuity (see e.g., Fig. 3a). As the step geometry is a recurring feature of all contact angle patterns considered, the step metastability limit will be used as a reference value in the remaining sections.

\subsection{Wedges}

The second-most elementary surface pattern is a wedge geometry as sketched in Fig. 2a with contact angle distribution

$\theta(x, y)=\left\{\begin{array}{ll}\theta_{0} & \text { if } x<0 \\ 0 & \text { else. }\end{array}\right.$ and $\quad\left|\arctan \left(\frac{y}{x}\right)\right|<\frac{\varphi}{2}$

Figure $4 a, b$ shows the conformation of a liquid layer shortly before nucleation of a dry-spot in the vicinity of hydrophobic corners with opening angles $\varphi=90^{\circ}$ and $270^{\circ}$. For $\varphi<180^{\circ}$, a single dry-spot nucleates first in the vicinity of the corner apex, whereas for $\varphi>180^{\circ}$, a 

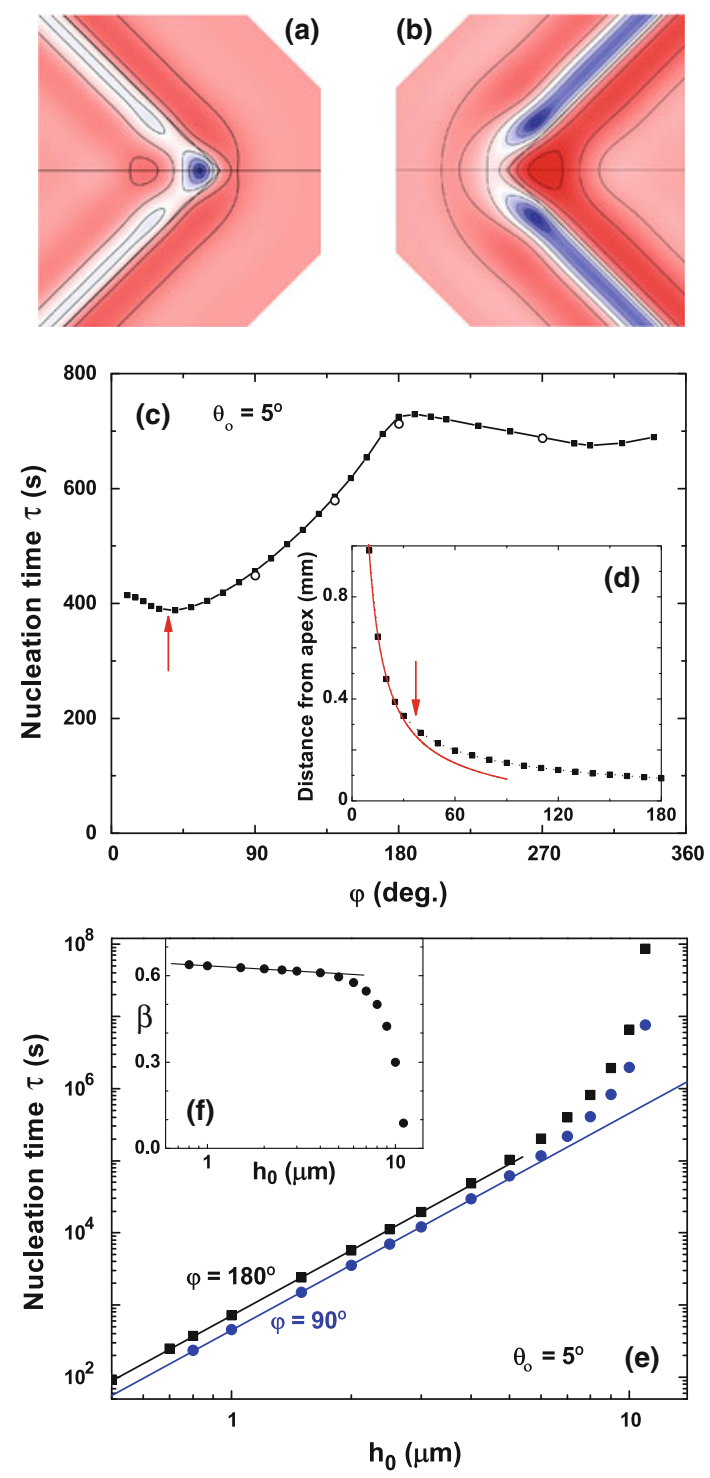

Fig. 4 a, b [Color onlilne] Contour plots of the liquid height profiles near a corner with opening angle $\varphi=90^{\circ}$ (a) and $270^{\circ}$ (b) shortly before nucleation of a dry-spot. Blue regions correspond to depressions, red ones to elevations of the height profile, pink ones to $h \approx h_{0}$. c Nucleation time near a corner as a function of $\varphi$ for $h_{0}=1 \mu \mathrm{m}$ and $\left(\theta_{\mathrm{o}}, n, m\right)=\left(5^{\circ}, 3,2\right) \quad($ solid symbols $)$ or $\left(\theta_{\mathrm{o}}, n, m\right)=\left(24^{\circ}, 4,3\right)$ (open symbols). d Distance of the nucleation point from the apex of the corner for $\theta_{\mathrm{o}}=5^{\circ}$ and $h_{0}=1 \mu \mathrm{m}$. The red arrow indicates the approximate location of the minimum in c. e Nucleation time as a function of $h_{0}$ for $\varphi=90^{\circ}$ and $180^{\circ}$ and $\left(\theta_{0}, n, m\right)=\left(5^{\circ}, 3,2\right)$. f Nucleation speed-up factor $\beta \equiv \tau\left(\varphi=90^{\circ}\right) / \tau\left(180^{\circ}\right)$ as a function of $h_{0}$ for $\left(\theta_{\mathrm{o}}, n, m\right)=\left(5^{\circ}, 3,2\right)$

doublet of dry-spots is formed. In Fig. $4 \mathrm{c}$ is plotted the nucleation time $\tau$ as a function of $\varphi$ for $h_{0}=1 \mu \mathrm{m}$ and the parameter sets $\left(\theta_{\mathrm{o}}, n, m\right)=\left(5^{\circ}, 3,2\right)$ and $\left(24^{\circ}, 4,3\right)$. A pronounced decrease in $\tau$ is observed for decreasing values of $\varphi$ between $180^{\circ}$ and $40^{\circ}$. A shallow minimum occurs for a hydrophobic wedge with $\varphi \approx 40^{\circ}$, at which $\tau$ amounts to approximately half of the value at $\varphi=180^{\circ}$. For $\varphi>180^{\circ}$, the value of $\tau$ depends only weakly on $\varphi$.
Figure $4 \mathrm{~d}$ presents the distance of the dry-spot nucleation point from the corner apex for $\varphi<180^{\circ}$. The solid line corresponds to the fit-function $d=\frac{0.24 \Lambda}{2 \tan \varphi / 2}$, which is an excellent representation of $d(\varphi)$ for small values of $\varphi$. Figure $4 \mathrm{f}$ compares $\tau$ as a function of $h_{0}$ for both $\varphi=90^{\circ}$ and $180^{\circ}$ for $\left(\theta_{0}, n, m\right)=\left(5^{\circ}, 3,2\right)$. The solid lines correspond to power law relations $\tau \sim h_{0}^{3}$. Figure $4 \mathrm{e}$ presents the dependence of the speed-up factor $\beta \equiv \tau(\varphi=$ $\left.90^{\circ}\right) / \tau\left(\varphi=180^{\circ}\right)$ on $h_{0}$. It remains nearly constant until $h_{0}$ reaches a value of $10 \mu \mathrm{m}$, upon which $\beta$ strongly decreases. This indicates a potential for more significant time-savings regarding dry-spot nucleation if $h_{0}$ is close to the metastability limit $h_{\text {us }}$.

Dry-spot nucleation is accelerated for $\varphi<180^{\circ}$ because liquid near the nucleation point is attracted towards the hydrophilic region along two directions. The height profile around the nucleation point in Fig. 4a has curvatures of comparable magnitude in both the $x$ and $y$ direction. For smaller values $\varphi \lesssim 40^{\circ}$, however, capillary effects retard the formation of a local depression in the vicinity of the apex. In addition, the height profile around the nucleation point is less circular and elongated along the hydrophilic/phobic boundaries for $\varphi \lesssim 40^{\circ}$. Consequently, more liquid has to be removed to reach a comparable dip amplitude, which causes the nucleation time to increase slightly.

Far from the apex, the wedge can locally be considered a hydrophobic stripe with almost parallel edges, if $\varphi$ is sufficiently small. In this case, a dry-spot will form at a position where the local width $w_{\text {min }}$ induces nucleation in the shortest time, i.e., at the minimum of the function $\tau(w)$. As a consequence, the nucleation time approaches a constant value in the limit $\varphi \rightarrow 0$. The nucleation point, however, moves further away from the apex (Fig. 4d) and is expected to coincide with $d=\frac{w_{\min }}{2 \tan \varphi / 2}$. We will show in Sect. 4 that $w_{\min } \approx 0.25 \Lambda$ in almost perfect agreement with the fitfunction in Fig. $4 \mathrm{~d}$.

In the same fashion as a step-like discontinuity in contact angles, a wedge pattern is also geometrically invariant under a transformation $(x, y) \rightarrow(x / a, y / a)$. Consequently, the equivalent argument as presented in Sect. 3.1 applies and a scaling $\tau \sim h_{0}^{2 m-1}$ follows for the film thickness range $h^{*} \ll$ $h_{0} \ll h_{\text {us }}$, consistent with the results shown in Fig. 4e.

\subsection{Step discontinuities with corrugated edges}

Motivated by the faster dry-spot nucleation in the vicinity of corners found in the Sect. 3.6, we now explore whether and to what extent the nucleation along a long straight edge can be accelerated by corrugating the edge in the fashion illustrated in Fig. 2b. We consider a saw-tooth pattern of period $L$ and amplitude $A$ with corresponding contact angle distribution 


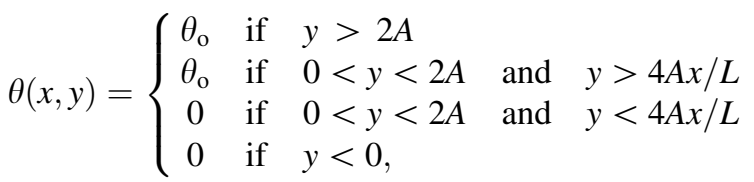

which is periodic in the $x$-direction $\theta(x, y)=\theta(x+L, y)$. Due to the existence of the lengthscale $L$, the geometric invariance of the step- and corner-geometry is absent, and
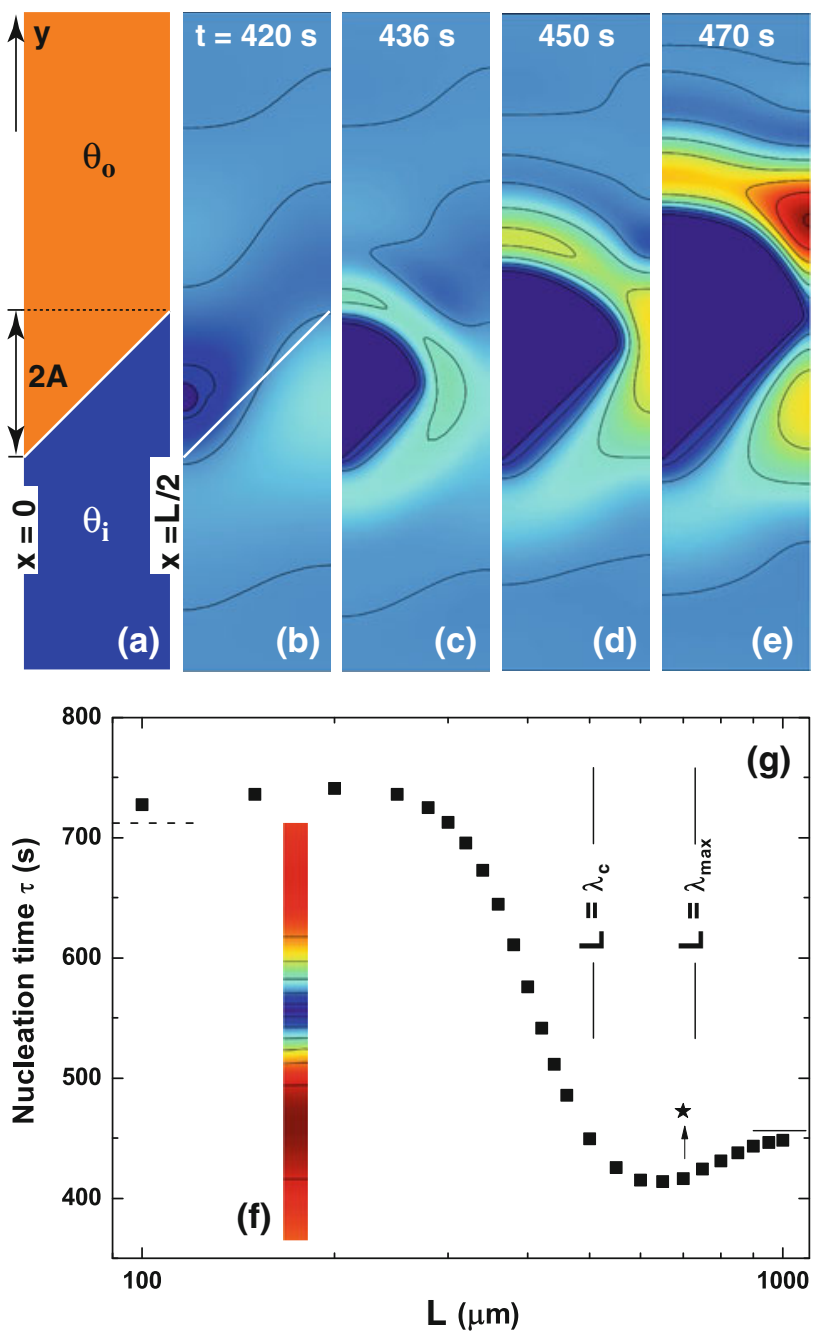

Fig. 5 a Sketch of half a period of contact angle discontinuity with corrugated edges with amplitude $A$ and wavelength $L$. b-e Contour plots of the liquid height profiles near a corrugated contact angle boundary with opening angle $\varphi=90^{\circ}$ at and after nucleation of a dryspot for $L=700 \mu \mathrm{m}, h_{0}=1 \mu \mathrm{m}$, and $\theta_{\mathrm{o}}=5^{\circ}$. f Contour plot of the liquid height profile for $L=100 \mu \mathrm{m}, h_{0}=1 \mu \mathrm{m}, \theta_{o}=5^{\circ}$ and $\varphi=$ $90^{\circ}$. g Dependence of nucleation time on the corrugation wavelength. The asterisk denotes the instant when the lateral extent of the dry-spot spans the entire computational domain for the case of $L=700 \mu \mathrm{m}$ as shown in e. The horizontal, dashed and solid lines correspond to the nucleation times of a straight edge and of a wedge with opening angle $\varphi=90^{\circ}$, taken from Fig. $3 \mathrm{c}$, respectively. The vertical lines correspond to $L=\lambda_{\max }=726.3 \mu \mathrm{m}$ and $L=\lambda_{\mathrm{c}}=\lambda_{\max } / \sqrt{2}$ the dimensionless ratio $L / \lambda_{\max }$ will qualitatively affect the solution.

In Fig. 5b-e, we show the time evolution of a growing dry-spot for $L=700 \mu \mathrm{m}$ and a corrugation angle $\varphi=90^{\circ}$, i.e., for the case $A=L / 2$. The dry-spot nucleates at $x=0$ in the vicinity of the inner corner. The dry-spot initially grows radially until it meets the hydrophobic-hydrophilic boundary (indicated by the white lines in Fig. 5a, b), after which it continues to expand until the entire corrugated edge is dewetted at $t=t_{*}$. In Fig. 5f, a contour plot of the height profile for $L=100 \mu \mathrm{m}$ is shown. In this case, the contour lines are to a good approximation parallel to the $x$-axis, i.e., essentially unaffected by the corrugated boundary shape, and the dry-spot nucleation occurs basically simultaneously along the entire edge. In Fig. 5g, we present the nucleation time $\tau$ as a function of corrugation wavelength $L$ for $h_{0}=1 \mu \mathrm{m}, \theta_{\mathrm{o}}=5^{\circ}$ and $\varphi=90^{\circ}$. The horizontal, dashed and solid lines correspond to the nucleation times $\tau_{\text {step }}$ of a straight edge and $\tau_{\text {wedge }}$ of a wedge with opening angle $\varphi=90^{\circ}$, taken from Fig. 3c, respectively. The vertical lines correspond to $L=\lambda_{\max }=726.3 \mu \mathrm{m}$ and $L=\lambda_{\mathrm{c}}=$ $\lambda_{\max } / \sqrt{2}$. The asterisk denotes the instant when the lateral width of the dry-spot spans the entire computational domain for the case of $L=700 \mu \mathrm{m}$ as shown in Fig. 5e. We expect that the hole expansion time $t_{*}-\tau$ increases with increasing wavelength, as the nucleation sites are further apart. Therefore, at very large wavelengths $t_{*}$ may exceed $\tau_{\text {step }}$.

A transition in the nucleation time $\tau$ from the value corresponding to a straight edge to the value corresponding to an infinite wedge is observed approximately at $L=\lambda_{\text {c }}$. The nucleation time exceeds $\tau_{\text {step }}$ for $L \lesssim 300 \mu \mathrm{m}$ and falls below $\tau_{\text {wedge }}$ for $L \gtrsim 500 \mu \mathrm{m}$. We attribute the first effect to a slight corrugation of the curve along which the initial height perturbation grows. The undershoot is attributed to a resonance of the corrugation wavelength with the maximally unstable wavelength of the linear instability mechanism applicable to homogeneous surfaces.

As can be seen in Fig. 4 f, the ratio $\tau_{\text {wedge }} / \tau_{\text {step }}$ strongly decreases when $h_{0}$ approaches the metastability limit, thereby increasing the time benefit gained from corrugating the step. Moreover, the speed of hole expansion is to good approximation independent of film thickness (Redon et al. 1991). Since $\tau$ increases with $h_{0}$, but $t_{*}-\tau$ remains approximately constant, the time needed for the lateral coalescence of the dry-spots becomes progressively insignificant in this regime.

\subsection{Isolated circles and stripes}

The most elementary isolated patterns with finite dimensions are a stripe of width $w_{\mathrm{o}}$ and corresponding contact angle distribution 
$\theta(x)= \begin{cases}\theta_{0} & \text { if } \quad|x| \leq \frac{w_{0}}{2} \\ 0 & \text { else }\end{cases}$

and a circle of radius $R_{\mathrm{o}}$ and corresponding contact angle distribution

$\theta(r)=\left\{\begin{array}{cl}\theta_{\mathrm{o}} & \text { if } r=\sqrt{x^{2}+y^{2}} \leq R_{\mathrm{o}} \\ 0 & \text { else. }\end{array}\right.$

Because of the axisymmetric nature of this distribution, we simulate the thin film evolution along a radial crosssection. Although, the final liquid conformation may not be axisymmetric for large circles $R \gg \lambda_{\text {max }}$, we expect that the film evolution up to nucleation is axisymmetric. The axisymmetric counterparts of Eqs. 1 and 2 are given by

$\frac{\partial h}{\partial t}-\frac{1}{r} \frac{\partial}{\partial r}\left(r \frac{h^{3}}{3 \mu} \frac{\partial p}{\partial r}\right)=0$

$p=-\frac{\gamma}{r} \frac{\partial}{\partial r}\left(r \frac{\partial h}{\partial r}\right)+\rho g h-\Pi$.

Figure 6 shows exemplary film thickness profiles $h(x, t)$ for different values of the $h_{0}$ on a substrate with a partially wetting stripe with $w_{\mathrm{o}}=0.4 \mathrm{~mm}$. In very thin films (Fig. 6a), a dry-spot is nucleated close to the hydrophilic/-phobic boundary, from which a dewetting front propagates leftwards over the hydrophobic domain. Due to the predominance of disjoining pressure in this regime, more dry-spots are nucleated leading to a cascade of droplets that are essentially immobilized and remain on the hydrophobic region. Given enough time, however, these droplets will coarsen and eventually disappear (Glasner and Witelski 2003) as most of the liquid is slowly redistributed through the precursor layer towards the hydrophilic region.

Thin films (Fig. 6b) and films of "intermediate" thickness (Fig. 6c) nucleate further from the hydrophilic/-phobic boundary, leading to the formation of a single droplet (Fig. 6b) or to complete clearing of the hydrophobic domain (Fig. 6c), which is typically the desired final state for technological applications. Very thick films (Fig. 6d) are (meta)stable towards dry-spot nucleation.

Figure 7 shows nucleation times as a function of $\theta_{\mathrm{o}}$ and $h_{0}$ for circles of $2 R_{\mathrm{o}}=0.4 \mathrm{~mm}$ (circles) and stripes of $w_{\mathrm{o}}=$ $0.4 \mathrm{~mm}$ (squares), respectively. The solid lines correspond to power law relations of the form $\tau \sim\left(1-\cos \theta_{\mathrm{o}}\right)^{-2} h_{0}^{2 m-1}$. The dashed and dotted lines indicate exemplary metastability limits for stripes and circles, respectively. The dashdotted line in Fig. 7b corresponds to the morphological transition from a clean hydrophobic pattern (Fig. 6c) to a single residual droplet (Fig. 6b) and satisfies $\tau_{\text {trans }} \sim h_{0}^{-3}$.

The transitions occur to good approximation when the geometric length scales $\left(2 R_{\mathrm{o}}, w_{\mathrm{o}}\right)$ equal $\frac{1}{2} \lambda_{\max }$, at which the local minimum of the height perturbation due to the hydrophobic-hydrophilic boundary is positioned in the center of the hydrophobic domain. The wiggles around these transitions reflect the resonance of the perturbation wavelength with the geometric length scale.
Fig. 6 Exemplary dewetting dynamics on an isolated stripe for different initial film thicknesses of a $h_{0}=0.1 \mu \mathrm{m}$, b $0.5 \mu \mathrm{m}$, c $2 \mu \mathrm{m}$, and d $20 \mu \mathrm{m}$, for $\theta_{\mathrm{o}}=6^{\circ}, w_{\mathrm{o}}=0.4 \mathrm{~mm}$ and parameter values $(n, m)=(3,2)$
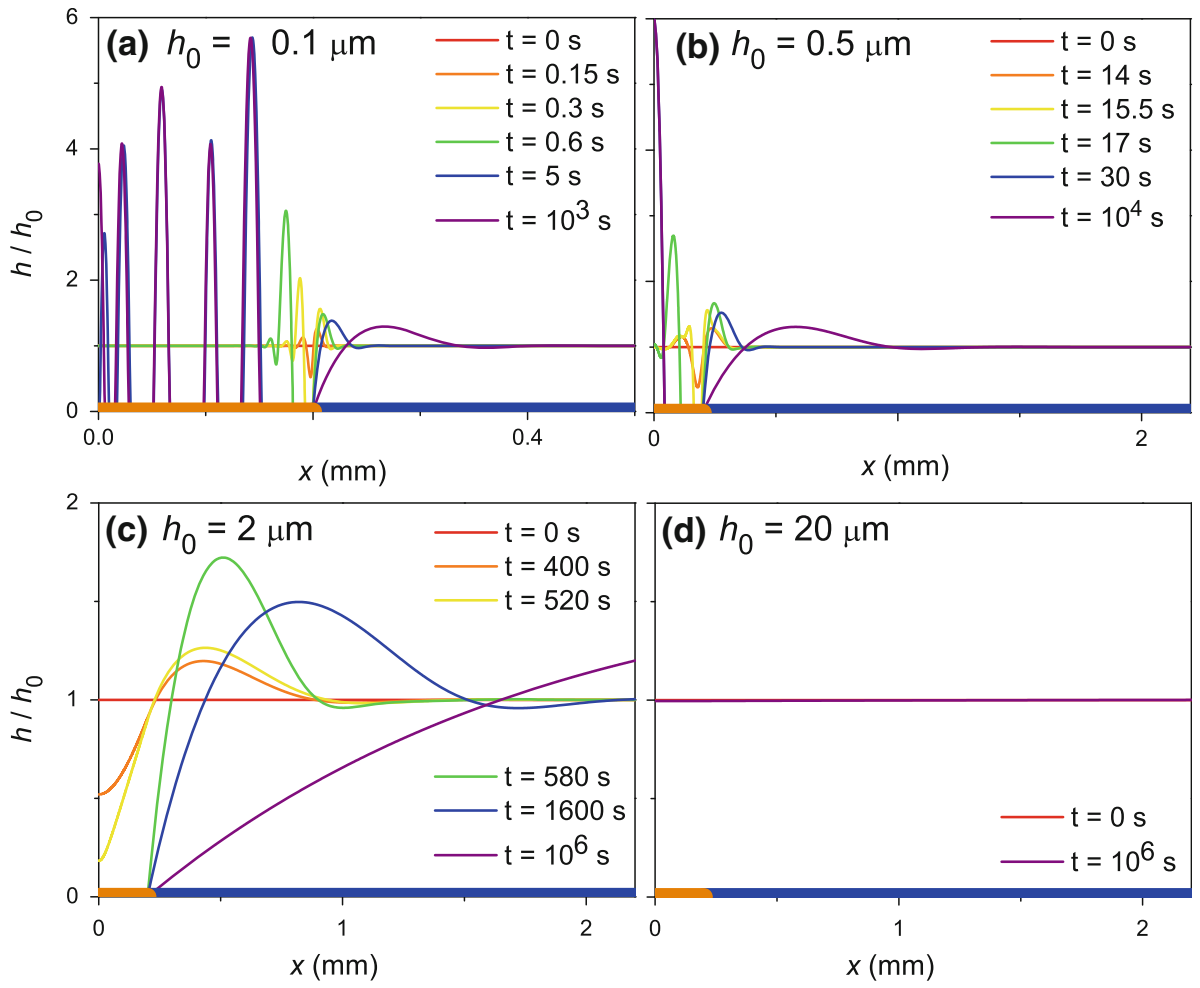
Fig. 7 Dry-spot nucleation time $\tau$ as a function of a contact angle $\theta_{\mathrm{o}}$ and $\mathbf{b}$ initial film thickness $h_{0}$ for $2 R_{\mathrm{o}}=w_{\mathrm{o}}=0.4 \mathrm{~mm}$ and parameter values $n=3$ and $m=2$

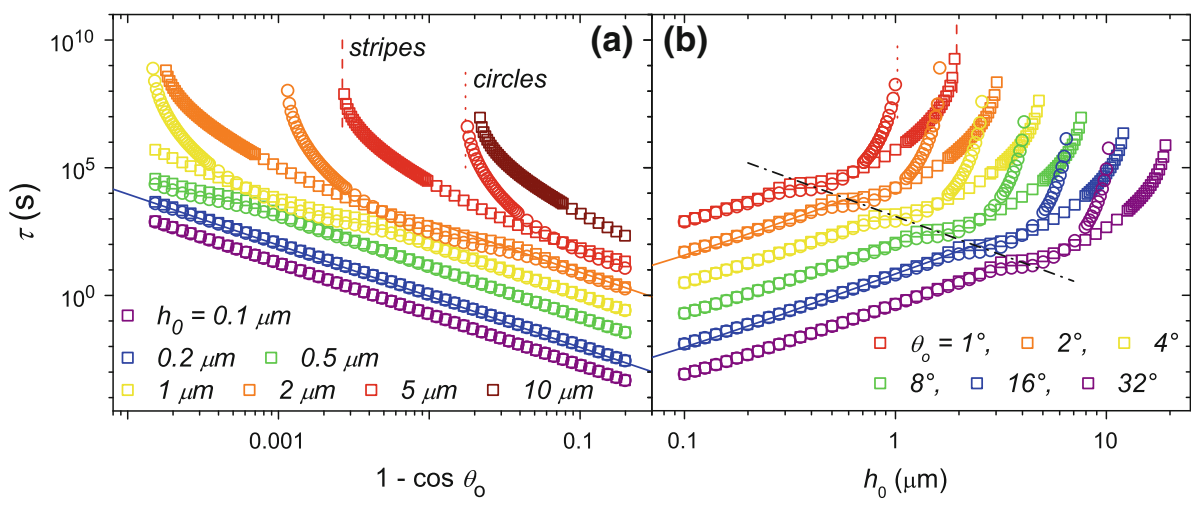

Far below the metastability limit, the nucleation times are identical for stripes and circles if $2 R_{\mathrm{o}}=w_{\mathrm{o}}$. The metastability limits of circles, however, correspond to higher values of $\theta_{\mathrm{o}}$ and smaller values of $h_{0}$ than for stripes, as a consequence of the higher capillary pressure for circles. This is a consequence of the fact that axisymmetric surfaces have curvatures in two directions.

The transitions from clean dewetting to a residual droplet are not significantly shifted, i.e., the span of the window of clean dewetting is smaller for circles than for stripes, as shown in Fig. 7b. This is also illustrated in the

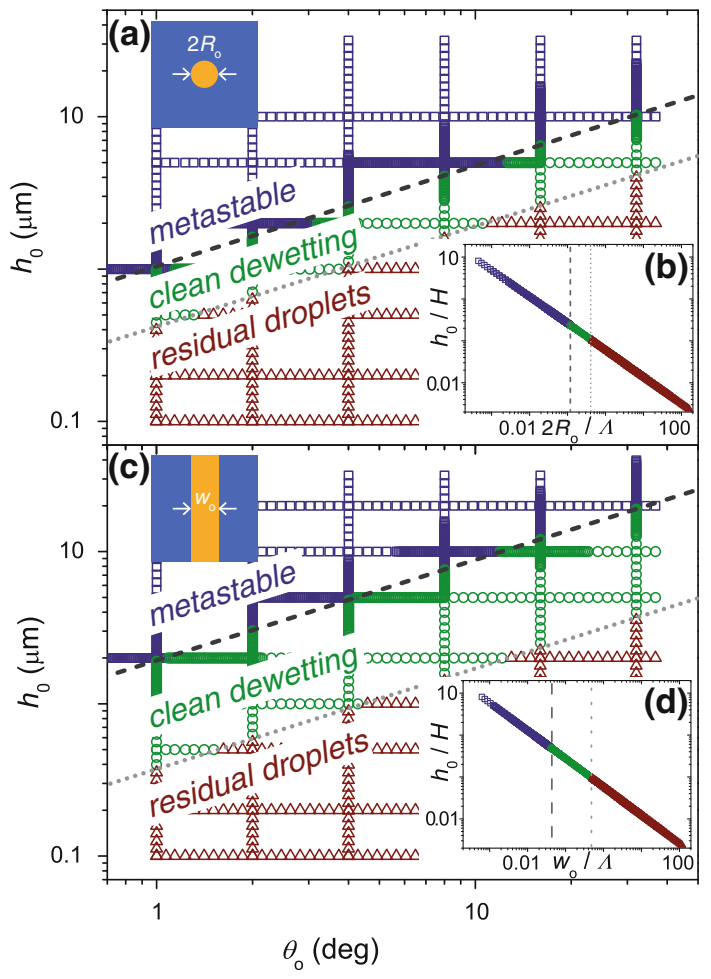

Fig. 8 Dimensional and non-dimensional morphology maps for $\mathbf{a}, \mathbf{b}$ circles of $2 R_{\mathrm{o}}=0.4 \mathrm{~mm}$ and c, d stripes of $w_{\mathrm{o}}=0.4 \mathrm{~mm}$ and parameter values $(n, m)=(3,2)$. The squares, circles, and triangles represent regimes I (metastable), II (clean dewetting), and III (residual droplets), respectively morphology maps of circles and stripes, which are shown in dimensional and non-dimensional form in Fig. 8. Three different regimes are considered: (I) metastable films that do not break up; (II) unstable films that leave no liquid on the hydrophobic region; and (III) unstable films that leave one or more droplets.

The chosen value of $2 R_{\mathrm{o}}=w_{\mathrm{o}}=0.4 \mathrm{~mm}$ is much smaller than the capillary length $\ell_{c}$, which indicates that capillary pressure will dominate over gravity as the stabilizing mechanism. Consequently, from a balance of disjoining pressure and capillary pressure, the transition between regimes I and II is expected to satisfy

$h_{0}=C\left(1-\cos \theta_{\mathrm{o}}\right)^{\frac{1}{m+1}}$.

The dashed dark gray and dotted light gray lines in Fig. 8 correspond to Eq. 17 with fitted prefactors $C$, which serve as excellent approximations for the boundaries of regime II, which typically is the desired process window. As a consequence, the regime boundaries collapse to unique values in the non-dimensional morphology maps. Thus, critical values of the confinement number $\mathrm{Co} \equiv$ $w_{\mathrm{o}} / \Lambda$ or $\mathrm{Co} \equiv 2 R_{\mathrm{o}} / \Lambda$ can be obtained, which are listed in Table 1.

Another important parameter in the design of the chemical pattern is the width of the hydrophobic stripe $w_{\mathrm{o}}$. Figure 9 shows nucleation times as a function of $w_{\mathrm{o}}$ and $h_{0}$ for stripes with a contact angle of $16^{\circ}$. Again, power-law relations have been derived considering a chemically homogeneous surface; they are represented by the solid lines. The dash-dotted lines in Fig. 9a represent the metastability limits. The dashed and dotted lines in Fig. 9a represent the global nucleation time minima and the

Table 1 Critical values of the confinement number for regime transitions, for $n=3$ and $m=2$

\begin{tabular}{lcc}
\hline Chemical pattern & $\mathrm{Co}_{\text {stable-clean }}$ & $\mathrm{Co}_{\text {clean-droplet }}$ \\
\hline Circle & $0.125 \pm 0.002$ & $0.42 \pm 0.01$ \\
Stripe & $0.04706 \pm 0.00006$ & $0.49 \pm 0.01$ \\
\hline
\end{tabular}



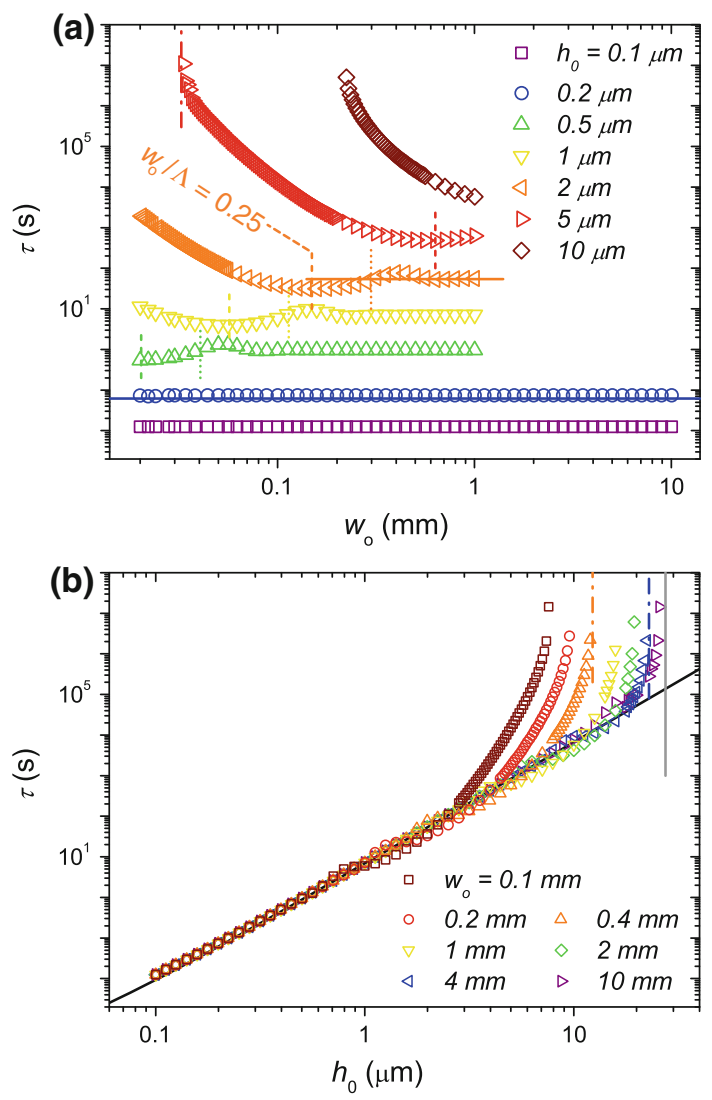

Fig. 9 Dry-spot nucleation times as a function of a the hydrophobic stripe width $w_{\mathrm{o}}$ and $\mathbf{b}$ the initial film thickness $h_{0}$, with a constant contact angle $\theta_{\mathrm{o}}=16^{\circ}$ and $(n, m)=(3,2)$

transition from clean dewetting to residual droplets, respectively. Using Eq. 9, one finds that this transition occurs for $w_{\mathrm{o}} \approx 0.49 \Lambda$ for all stripe widths considered. For large $w_{\mathrm{o}}$, the power-laws in Fig. $9 \mathrm{~b}$ become independent of $w_{\mathrm{o}}$ because the dynamics around the two stripe edges no longer interact with each other. For narrower stripes, the stabilizing influence of capillary pressure plays an increasingly important role and will slow down and eventually prevent dry-spot nucleation. Global minima in the nucleation time are found at $w_{\mathrm{o}} \approx 0.25 \Lambda$, which is in excellent agreement with the local width at which nucleation occurs for narrow wedges, as found in Sect. 2 for narrow wedges (Fig. 4d). The vertical, solid gray line in Fig. 9b illustrates the metastability limit for the step pattern, to which the family of curves converges for increasing $w_{\mathrm{o}}$.

Figure 10 illustrates the liquid configuration after dewetting as a function of $h_{0}$ and hydrophobic stripe width $w_{\mathrm{o}}$. The non-dimensional equivalent is shown in the inset. As in Fig. 8, the three types of morphologies I-III are indicated by squares, circles, and triangles. The gray dashed and dotted lines indicate the regime transitions. In addition, the step metastability limit $h_{0}=H_{\text {step }}$ is represented by the

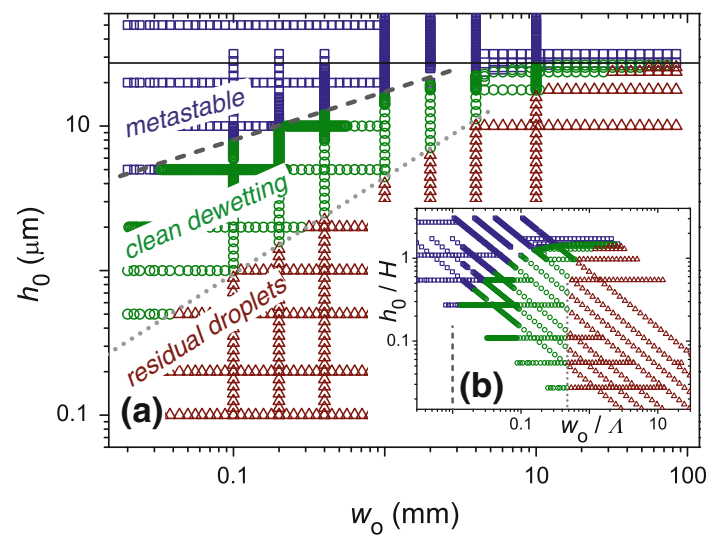

Fig. 10 Morphology maps for isolated hydrophobic stripes of varying width, with $\theta_{\mathrm{o}}=16^{\circ}$ and $(n, m)=(3,2)$. The squares, circles, and triangles represent regimes I (metastable), II (clean dewetting), and III (residual droplets), respectively

solid black line. The regime transition lines correspond to constant confinement numbers and are of the form $h_{0}=$ $\widehat{C} w_{\mathrm{o}}^{\frac{2}{m+1}}$, with $\widehat{C}$ a fitted prefactor. For small $h_{0}$ and $w_{\mathrm{o}}$, the regime transitions perfectly correspond to the fitted powerlaws, but for wider stripes, they asymptotically converge towards the step metastability limit. Similar to the nucleation dynamics, (Fig. 9b), the conformation becomes independent of $w_{\mathrm{o}}$ for wide stripes. As a consequence, the clean window decreases and eventually disappears.

\subsection{Influence of disjoining pressure isotherm}

In this section, we investigate the influence of the disjoining pressure on break-up behavior by varying the coefficients $n$ and $m$ in Eq. 3. As the attractive potential dominates over the repulsive potential during the initial state of perturbation growth, most attention is devoted to the influence of the coefficient $m$. Figure 11 shows exemplary thickness profiles for different values of $m$, for which the difference in exponents is kept constant: $n=m+1$. The thin film evolution was simulated on a $0.4 \mathrm{~mm}$ wide stripe with a contact angle $\theta_{\mathrm{o}}=8^{\circ}$, using initial thickness values adapted to the window of clean operation, estimated using Eq. 17.

Figure 11 reveals an interesting feature: whereas clean dewetting is obtained for $m>1.5$, one or more residual droplets are left behind for smaller values of $m$ in all cases considered. In contrast to Fig. 6a, b, where the center height of the residual droplet reaches approximately $5 h_{0}$, the residual droplets in Fig. 11a, b have very small volumes. For small values of $m$, the formation of these small droplets is preceded by the occurrence of a plateau around the center, which is, similarly to a very thin flat film, highly unstable to the disjoining pressure. For the parameter range considered, we did not observe small droplets for $m>1.75$. This transition approximately coincides with a qualitative change of the 
Fig. 11 Exemplary film profiles for different disjoining pressure isotherms

$(n, m)=\mathbf{a}(2.25,1.25), \mathbf{b}(4,3)$

for parameter values $\theta_{\mathrm{o}}=16^{\circ}$

and $w_{\mathrm{o}}=0.4 \mathrm{~mm}$

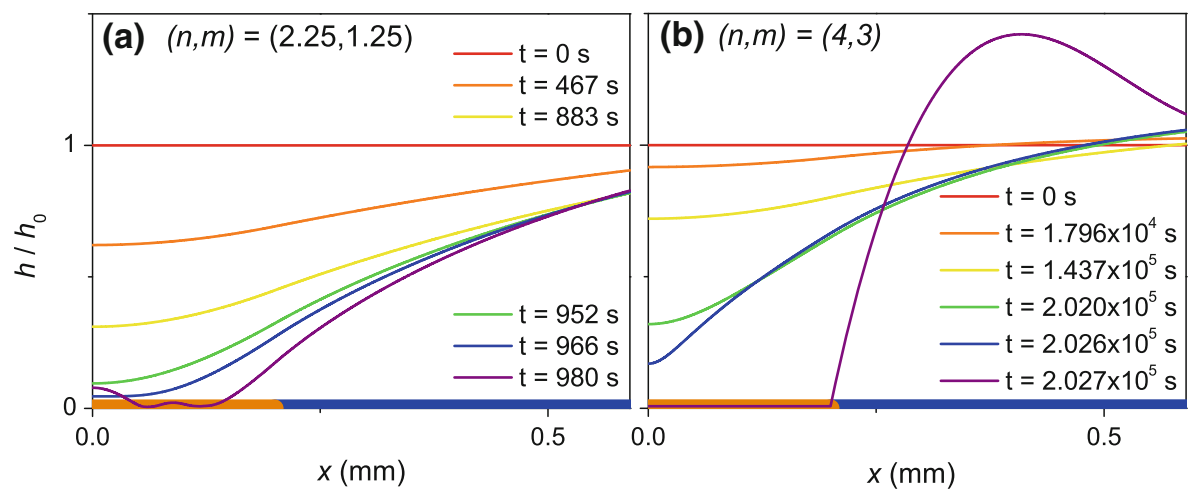

hydrodynamic flux term due to the attractive disjoining pressure component $h^{3} \frac{\mathrm{d}_{m}}{\mathrm{~d} x} \sim h^{3} / h^{m+1}$. When $3-(m+1) \geq 0$, i.e., $m \leq 2$, the flux no longer increases with decreasing film thickness. It is intuitive to assume that this feature is a requirement for clean dewetting. This simplified argument does not account for the influence of capillary pressure and the repulsive component of $\Pi_{\mathrm{SE}}$, which may explain why the transition occurs at a value slightly different from 2 .

Figure 12 shows the nucleation time as a function of $h_{0}$ for two different sets of values of $(n, m)$. In Fig. 12a, the influence of $m$ is shown, while maintaining $n=m+1$; Fig. $12 \mathrm{~b}$ illustrates the influence of $n$, for a constant $m=1.5$.

For sufficiently large $n-m$, the attractive component of $\Pi_{\mathrm{SE}}$ dominates the behavior for $h_{0} \gg h^{*}$. In line with the discussion in Sect. 1, a power-law behavior $\tau=K(n, m)$ $h_{0}^{2 m-1}$ is expected to hold, in which $K$ is a constant derived from Eq. 3. Conversely, for small values of $n-m$, the influence of the repulsive disjoining pressure component and thus the value of $n$ increases. Indeed, Fig. 12b shows that for larger values of $n-m$, the dependence of $\tau$ on $h_{0}$ in regime III converges towards the power-law $\tau \sim h_{0}^{2 m-1}$.

In Figure 13a, b, morphology maps as a function of $h_{0}, n$ and $m$ are presented for the same sets of $(n, m)$ values as in Fig. 12. We consider the occurrence of small droplets
(Fig. 11a, b) as the equivalent of the clean dewetting regime, which is strictly observed only for $m>1.75$. Droplets were considered small if their maximum thickness $h_{\mathrm{d}}$ was less than $\frac{1}{4} h_{0}$ and large if $h_{\mathrm{d}}>h_{0}$. Morphologies where $\frac{1}{4} h_{0} \leq h_{\mathrm{d}} \leq h_{0}$ were deemed inconclusive and are therefore not included in the morphology maps. As a reference, the values of $h_{\text {us }}$ have been added as black solid lines. The dashed dark gray and dotted gray lines correspond to $C_{1,2} h_{\mathrm{us}}$, with fit parameters $C_{1}<C_{2}<1$, which demarcate the regime transitions relatively accurately.

\subsection{Arrays of infinitely long stripes}

Whereas in the previous section we considered dry-spot nucleation induced by single isolated stripes, we now focus on stripe arrays. The width of the hydrophilic stripe $w_{\mathrm{i}}$ constitutes a second geometric length scale. In Figure 14a, nucleation times are presented as a function of $h_{0}$ for various $w_{\mathrm{i}}$, a constant value of $w_{\mathrm{o}}=0.4 \mathrm{~mm}$ and $\theta_{\mathrm{o}}=4^{\circ}, 8^{\circ}, 16^{\circ}$. The black lines represent the data for an isolated hydrophobic stripe of the same width, which are added as a reference case, corresponding to the limit $w_{\mathrm{i}} / w_{\mathrm{o}} \rightarrow \infty$. In the limit of very thin films, $\tau$ is independent of $w_{\mathrm{i}}$. For intermediate $h_{0}$, a smaller value of $w_{\mathrm{i}}$ implies that liquid redistributed from the hydrophobic to
Fig. 12 Nucleation times versus initial thickness for a varying $m$ with $n=m+1$ and b varying $n$ with $m=1.5$. $w_{\mathrm{o}}=0.4 \mathrm{~mm}$ for $\theta_{\mathrm{o}}=8^{\circ}$ and $w_{\mathrm{o}}=0.4 \mathrm{~mm}$

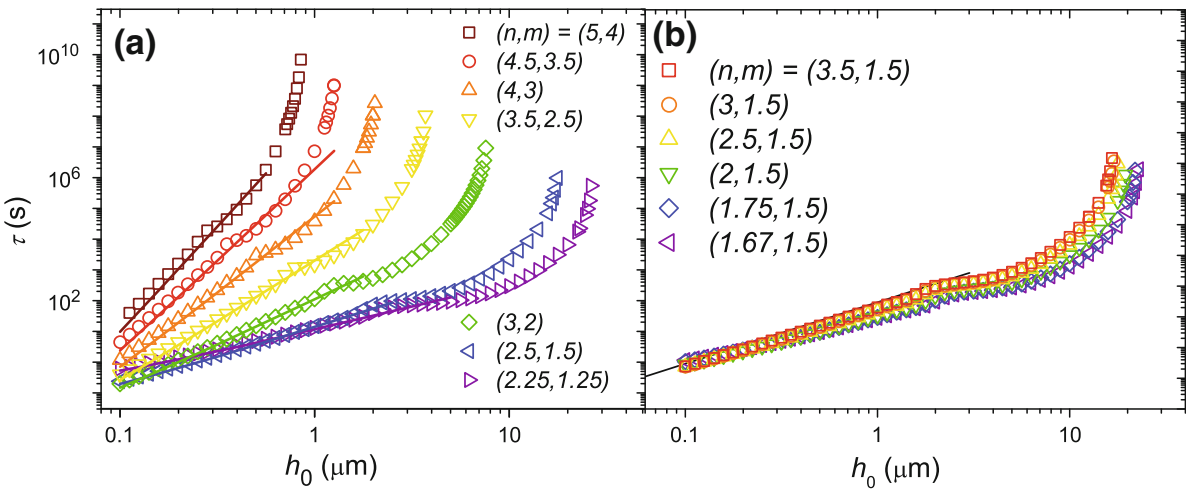


Fig. 13 Morphology maps for a varying $m$ with $n=m+1$ and $\mathbf{b}$ varying $n$ with $m=1.5$ for $\theta_{\mathrm{o}}=8^{\circ}$ and $w_{\mathrm{o}}=0.4 \mathrm{~mm}$. The squares, circles, and triangles represent regimes I (metastable), II (clean dewetting), and III (residual droplets), respectively; the hexagons represent the equivalent of regime II

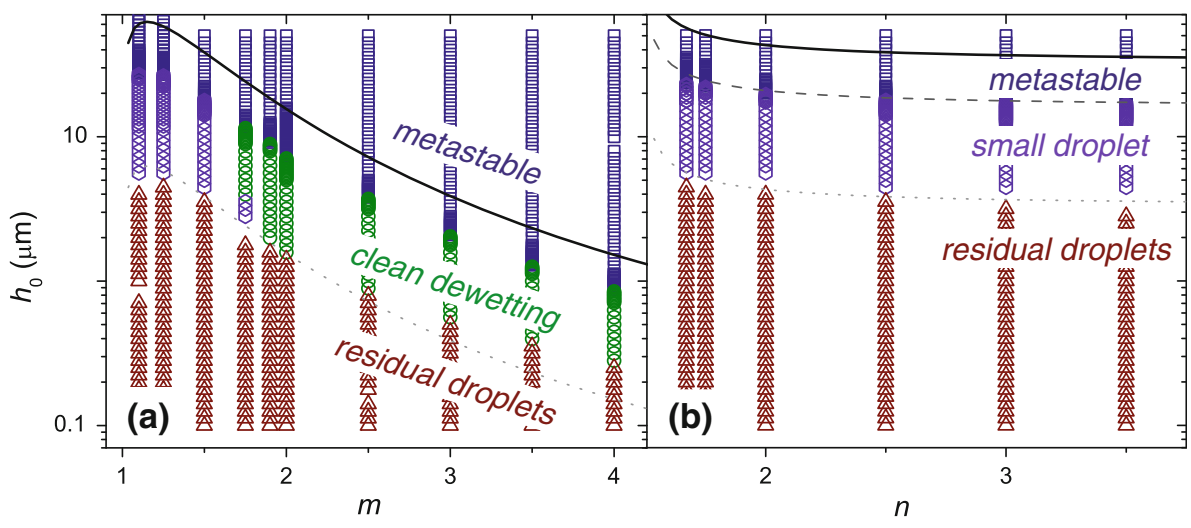

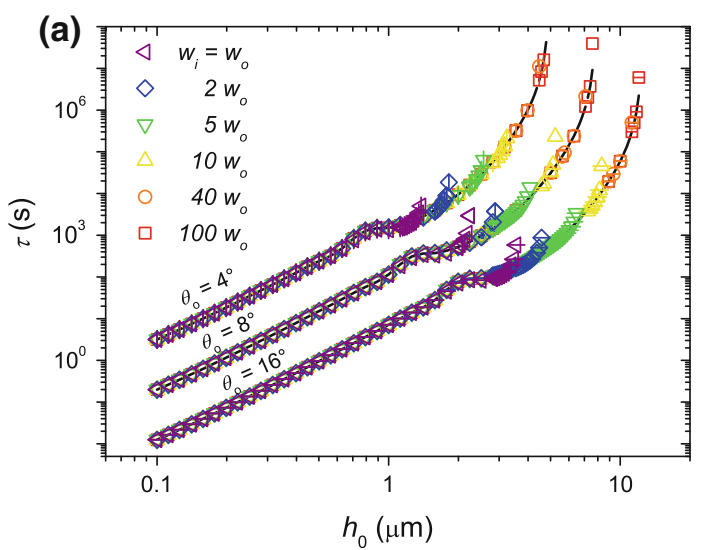

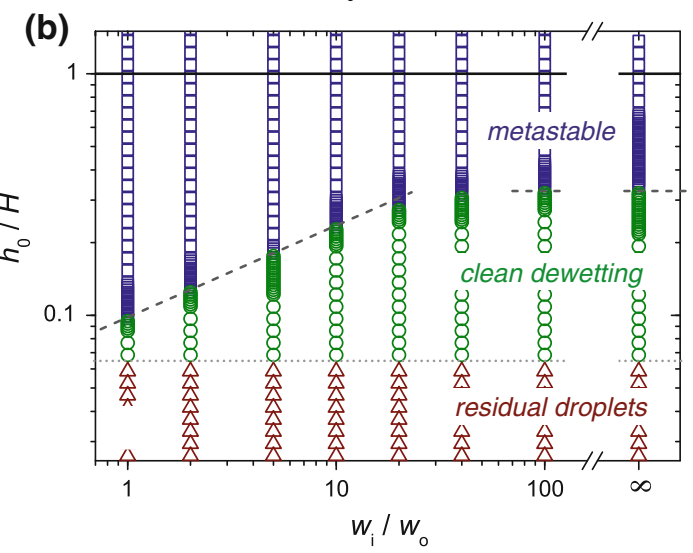

Fig. 14 a Nucleation times versus initial thickness for different hydrophilic stripe widths for $\theta_{\mathrm{o}}=4^{\circ}, 8^{\circ}, 16^{\circ}$ and $\mathbf{b}$ morphology map as a function of $h_{0} / H$ and $w_{\mathrm{i}} / w_{\mathrm{o}}$ for a periodic stripe array, $w_{\mathrm{o}}=0.4 \mathrm{~mm}$ and $(n, m)=(3,2)$. The squares, circles, and triangles in b represent regimes I (metastable), II (clean dewetting), and III (residual droplets), respectively

the hydrophilic stripes induces larger increase in film thickness. Consequently, the capillary pressure on the hydrophilic stripe increases, which provides a larger opposing force to the growth of the perturbation. Smaller values of $w_{\mathrm{i}}$ therefore shift the metastability limit towards smaller values of $h_{0}$, as is indeed observed in Fig. 14a. Interestingly, the transition from morphology regime III to
II is unaffected by $w_{\mathrm{i}}$ in the cases considered. The $w_{\mathrm{i}}$ dependence of the metastability limit also appears in the morphology map, which is shown in Fig. 14b as a function of $h_{0} / H$ and $w_{\mathrm{i}} / w_{\mathrm{o}}$. The morphologies for an isolated hydrophobic stripe are shown above the abscissa label $\infty$ as a reference case. The dashed dark gray and dotted gray lines mark the morphological regime transitions and serve as guides to the eye. The metastability limit in the interval $1 \leq w_{\mathrm{i}} / w_{\mathrm{o}} \leq 10$ is well approximated by a power-law of the form $h_{0} \sim w_{\mathrm{i}}^{0.4}$.

In agreement with the observations from Fig. 14a, the transition from residual droplets to clean dewetting does not depend on the hydrophilic stripe width as long as $w_{\mathrm{i}} \geq w_{\mathrm{o}}$, whereas the metastability limit does depend on $w_{\mathrm{i}}$. As a consequence, the window of clean operation is significantly decreased for narrower hydrophilic stripes. Corresponding to the convergence of the metastability limits observed in Fig. 14a, the metastability limit becomes indistinguishable from the isolated stripe value for $w_{\mathrm{i}} / w_{\mathrm{o}}>40$.

\section{Summary and conclusions}

Sufficiently thin films on partially wetting, chemically homogeneous surfaces are unstable and nucleate dry-spots. When their thickness exceeds the metastability limit, they become linearly stable towards infinitesimal perturbations. We have characterized the influence of various chemical surface heterogeneities on the metastability limits and nucleation times of thin liquid films.

The most elementary patterns are two adjacent halfplanes of different contact angle. The contact angle transition leads to a discontinuous disjoining pressure, which induces a localized perturbation of non-infinitesimal amplitude. Therefore, films can nucleate a dry-spot even for thicknesses that are metastable on homogeneous surfaces and the metastability limit shifts towards larger thickness values. 
Wedge-shaped contact angle patterns can significantly accelerate dry-spot nucleation. Moreover, saw-tooth shaped corrugations of a step edge lead to a decrease of the nucleation time. They exhibit a resonance when the corrugation wavelength corresponds to the maximally unstable perturbation wavelength for a homogeneously hydrophobic region.

Isolated hydrophobic patterns of finite dimensions are subject to confinement effects, when the geometric lengthscale and the maximally unstable wavelength are of comparable magnitude. The stabilizing influence of the confinement-induced capillary pressure leads to a shift of the metastability limit towards thinner films. For small hydrophobic circles, this effect is stronger than for hydrophobic stripes, since axisymmetric perturbations have curvatures in two directions and thus higher capillary pressures. The metastability limit for isolated, finite patterns approaches the value corresponding to two adjacent half-planes for increasing pattern size. In periodic stripe arrays, the hydrophilic stripe width constitutes a second geometric lengthscale that further lowers the metastability limit.

In a certain thickness range, bounded by the metastability limit, dry-spot nucleation proceeds without the formation of a residual droplet on the hydrophobic pattern. This "clean" process window and its dependence on pattern dimensions and film thickness, provides valuable insight for e.g., coating technologies or solution-based manufacturing of organic electronic devices.

Acknowledgments This research forms part of the research programme of the Dutch Polymer Institute (DPI), project \#640. The authors wish to thank Juliane Gabel, Charlotte Kjellander, Ronn Andriessen, Jasper Michels, Maosheng Ren and Ike de Vries of the Holst Centre for the fruitful collaboration.

Open Access This article is distributed under the terms of the Creative Commons Attribution Noncommercial License which permits any noncommercial use, distribution, and reproduction in any medium, provided the original author(s) and source are credited.

\section{References}

Bergeron V, Radke CJ (1992) Equilibrium measurements of oscillatory disjoining pressures in aqueous foam films. Langmuir 8:3020-3026

Biebuyck HA, Whitesides GM (1994) Self-organization of organic liquids on patterned self-assembled monolayers of alkanethiolates on gold. Langmuir 10:2790-2793

Bower CL, Simister EA, Bonnist E, Paul K, Pightling N, Blake TD (2007) Continuous coating of discrete areas of a flexible web. AIChE J 53:1644-1657

Brasjen BJ, van Cuijk AW, Darhuber AA (2011) Dip-coating of chemically patterned surfaces. Chem Eng Process 50:565-568

Brochard Wyart F, Daillant J (1990) Drying of solids wetted by thin liquid films. Can J Phys 68:1084-1088
Brochard Wyart F, Martin P, Redon C (1993) Liquid/liquid dewetting. Langmuir 9:3682-3690

Brusch L, Kühne H, Thiele U, Bär M (2002) Dewetting of thin films on heterogeneous substrates: pinning versus coarsening. Phys Rev E 66:011602

Buguin A, Vovelle L, Brochard-Wyart F (1999) Shocks in inertial dewetting. Phys Rev Lett 83:1183-1186

Burelbach JP, Bankoff SG, Davis SH (1988) Nonlinear stability of evaporating/condensing liquid films. J Fluid Mech 195:463-494

Cahn JW (1965) Phase separation by spinodal decomposition in isotropic systems. J Chem Phys 42:93-99

Darhuber AA, Troian SM, Davis JM, Miller SM, Wagner S (2000) Selective dip-coating of chemically micropatterned surfaces. J Appl Phys 88:5119-5126

Darhuber AA, Troian SM, Wagner S (2001) Physical mechanisms governing pattern fidelity in microscale offset printing. J Appl Phys 90:3602-3609

Derjaguin BV, Churaev NV, Muller VM (1987) Surface forces. Consultants Bureau, New York

Fetzer R, Jacobs K, Münch A, Wagner B, Witelski TP (2005) New slip regimes and the shape of dewetting thin liquid films. Phys Rev Lett 95:127801

Finn R (1986) Equilibrium capillary surfaces. Springer Verlag, New York

Glasner KB, Witelski TP (2003) Coarsening dynamics of dewetting films. Phys Rev E 67:016302

Gumerman RJ, Homsy GM (1975) The stability of radially bounded thin films. Chem Eng Commun 2:27-36

Jain RK, Ruckenstein E (1976) Stability of stagnant viscous films on a solid surface. J Colloid Interface Sci 54:108-116

Julthongpiput D, Zhang W, Douglas JF, Karim A, Fasolka MJ (2007) Pattern-directed to isotropic dewetting transition in polymer films on micropatterned surfaces with differential surface energy contrast. Soft Matter 3:613-618

Kalpathy SK, Francis LF, Kumar S (2010) Shear-induced suppression of rupture in two-layer thin liquid films. J Colloid Interface Sci 348:271-279

Kao JC-T, Golovin AA, Davis SH (2006) Rupture of thin films with resonant substrate patterning. J Colloid Interface Sci 303:532-545

Kargupta K, Konnur R, Sharma A (2000) Instability and pattern formation in thin liquid films on chemically heterogeneous substrates. Langmuir 16:10243-10253

Kargupta K, Sharma A (2002) Dewetting of thin films on periodic physically and chemically patterned surfaces. Langmuir 18: 1893-1903

Kargupta K, Sharma A (2003) Mesopatterning of thin liquid films by Ttemplating on chemically patterned complex substrates. Langmuir 19:5153-5163

Kargupta K, Sharma A, Khanna R (2004) Instability, dynamics, and morphology of thin slipping films. Langmuir 20:244-253

Karthaus O, Gråsjö L, Maruyama N, Shimomura M (1999) Formation of ordered mesoscopic polymer arrays by dewetting. Chaos 9: 308-314

Kipphan H (ed) (2001) Handbook of print media. Springer, Berlin

Kissa E (ed) (2001) Fluorinated surfactants and repellents. Marcel Dekker, New York

Koch W, Dietrich S, Napiórkowski M (1995) Morphology and line tension of liquid films adsorbed on chemically structured substrates. Phys Rev E 51:3300-3317

Moilliet JL (ed) (1963) Waterproofing and water-repellency. Elsevier, Amsterdam

Moosavi A, Rauscher M, Dietrich S (2008) Motion of nanodroplets near chemical heterogeneities. Langmuir 24:734-742

Noblin X, Buguin A, Brochard-Wyart F (2006) Cascade of shocks in inertial liquid-liquid dewetting. Phys Rev Lett 96:156101 
Oron A, Davis SH, Bankoff SG (1997) Long-scale evolution of thin liquid films. Rev Mod Phys 69:931-980

Pototsky A, Bestehorn M, Thiele U (2004) Control of the structuring of thin soft matter films by means of different types of external disturbance. Physica D 199:138-148

Redon C, Brochard Wyart F, Rondelez F (1991) Dynamics of dewetting. Phys Rev Lett 66:715-718

Reiter G (1992) Dewetting of thin polymer films. Phys Rev Lett 68:75-78

Reiter G (1993) Unstable thin polymer films: rupture and dewetting processes. Langmuir 9:1344-1351

Reiter G, Hamieh M, Damman P, Sclavons S, Gabriele S, Vilmin T, Raphaël E (2005) Residual stresses in thin polymer films cause rupture and dominate early stages of dewetting. Nat Mater 4: 754-758

Ruckenstein E, Jain RK (1974) Spontaneous rupture of thin liquid films. J Chem Soc Faraday Trans 270:132-147

Schwartz LW, Eley RR (1998) Simulation of droplet motion on lowenergy and hetergeneous surfaces. J Colloid Interface Sci 202: 173-188

Seemann R, Herminghaus S, Jacobs K (2001) Dewetting patterns and molecular forces: a reconciliation. Phys Rev Lett 86:5534-5537

Sehgal A, Ferreiro V, Douglas JF, Amis EJ, Karim A (2002) Patterndirected dewetting of ultrathin polymer films. Langmuir 18: 7041-7048

Senefelder A (1911) The invention of lithography. Fuchs \& Lang Manufact Comp, New York

Sharma A (1993) Relationship of thin film stability and morphology to macroscopic parameters of wetting in the apolar and polar systems. Langmuir 9:861-869

Sharma A (1998) Stability and breakup of thin evaporating water films: role of hydrophobic interaction. J Colloid Interface Sci 199: 212-214

Sharma A, Ruckenstein E (1986) Finite-amplitude instability of thin free and wetting films: prediction of lifetimes. Langmuir 2: 480-494

Sharma A, Jameel AT (1993) Nonlinear stability, rupture, and morphological phase separation of thin liquid films on apolar and polar substrates. J Colloid Interface Sci 161:190-208

Sharma A, Kargupta K (2003) Instability and dynamics of thin slipping films. Appl Phys Lett 83:3549-3551
Sharma A, Mittal J, Verma R (2002) Instability and dewetting of thin films induced by density variations. Langmuir 18:10213-10220

Sharma A, Konnur R, Kargupta K (2003) Thin liquid films on chemically heterogeneous substrates: self-organization, dynamics and patterns in systems displaying a secondary minimum. Physica A 318:262-278

Sheludko A (1967) Thin liquid films. Adv Colloid Interface Sci $1: 391-464$

Snoeijer JH (2006) Free surface flows with large slopes: beyond lubrication theory. Phys Fluids 18:021701

Stöckelhuber KW (2003) Stability and rupture of aqueous wetting films. Eur Phys J E 12:431-435

Stöckelhuber KW, Radoev B, Wenger A, Schulze HJ (2004) Rupture of wetting films caused by nanobubbles. Langmuir 20:164-168

Thiele U, Velarde MG, Neuffer K (2001a) Dewetting: film rupture by nucleation in the spinodal regime. Phys Rev Lett 87:016104

Thiele U, Velarde MG, Neuffer K, Pomeau Y (2001b) Film rupture in the diffuse interface model coupled to hydrodynamics. Phys Rev E 64:031602

Thiele U, Brusch L, Bestehorn M, Bär M (2003) Modelling thin-film dewetting on structured substrates and templates: bifurcation analysis and numerical simulations. Eur Phys J E 11:255-271

Verma R, Sharma A, Banerjee I, Kargupta K (2006) Spinodal instability and pattern formation in thin liquid films confined between two plates. J Colloid Interface Sci 296:220-232

Vrij A (1966) Possible mechanism for the spontaneous rupture of thin, free liquid films. Discuss Faraday Soc 42:23-33

Vrij A, Th J, Overbeek G (1968) Rupture of thin liquid films due to spontaneous fluctuations in thickness. J Am Chem Soc 90: 3074-3078

Williams MB, Davis SH (1982) Nonlinear theory of film rupture. J Colloid Interface Sci 90:220-228

Xie R, Karim A, Douglas JF, Han CC, Weiss RA (1998) Spinodal dewetting of thin polymer films. Phys Rev Lett 81:1251-1254

Zhang Z, Wang Z, Xing R, Han Y (2003) Patterning thin polymer films by surface-directed dewetting and pattern transfer. Polymer 44:3737-3743

Zhu D, Li X, Zhang G, Zhang X, Zhang X, Wang T, Yang B (2010) Mimicking the rice leaf-from ordered binary structures to anisotropic wettability. Langmuir 26:14276-14283 